\title{
ANÁLISE BIBLIOMÉTRICA DA PRODUÇÃO DISCENTE DOS CURSOS DE PÓS- GRADUAÇÃO DO INSTITUTO DE MATEMÁTICA DA UFRJ
}

\section{BIBLIOMETRIC ANALYSIS OF DISCENT PRODUCTION OF POSTGRADUATE COURSES OF THE INSTITUTE OF MATHEMATICS OF UFRJ}

\author{
Marília Cossich Ramos \\ Mestre em Biblioteconomia \\ Bibliotecária do Instituto de Matemática da UFRJ \\ Biblioteca da Pós-Graduação em Matemátca \\ mariliacossich@gmail.com
}

Resumo

\begin{abstract}
Apresenta as teses e dissertações produzidas pelos alunos dos cursos de Pósgraduação do Instituto de Matemática da Universidade Federal do Rio de Janeiro. Tem por objetivo mapear a produção científica dos últimos dez anos dos discentes dos Programas de pós-graduação do Instituto de Matemática da Universidade Federal do Rio de Janeiro. A metodologia utilizada foi uma pesquisa descritiva de caráter quantitativo por amostragem. O universo foram os cursos dos Programas de pósgraduação do Instituto de Matemática da Universidade Federal do Rio de Janeiro. A coleta dos dados foi realizada na base bibliográfica da universidade, a Base Minerva, a fim de se obter o número de teses e dissertações produzidas e somente aquelas que foram depositadas na Biblioteca Leopoldo Nachbin. Os resultados obtidos foram os números de teses e dissertações referentes à produção dos alunos dos cursos da pósgraduação cujos trabalhos foram depositados na Biblioteca Leopoldo Nachbin. Conclui ressaltando a importância da Biblioteca Digital de Teses e Dissertações para a divulgação e popularização da ciência.
\end{abstract}

Palavras-chave: Educação. Programas de pós-graduação. Bibliotecas universitárias.

\begin{abstract}
It presents the theses and dissertations produced by the students of the Postgraduate courses of the Institute of Mathematics of the Federal University of Rio de Janeiro. It aims to map the scientific production of the last ten years of students of the graduate programs of the Institute of Mathematics at the Federal University of Rio de Janeiro. The methodology used was a quantitative descriptive research by sampling. The universe consisted of courses in the graduate programs of the Institute of Mathematics of the Federal University of Rio de Janeiro. Data collection was performed on the university's bibliographic base, Base Minerva, in order to obtain the number of theses and dissertations produced and only those that were deposited at the Leopoldo Nachbin Library. The results obtained were the number of theses and dissertations related to the production of students of graduate courses whose works were deposited at the Leopoldo Nachbin Library. It concludes by emphasizing the importance of the Digital Library of Theses and Dissertations for the dissemination and popularization of science.
\end{abstract}

Keywords: Education. Postgraduate programs. University libraries. 


\section{INTRODUÇÃO}

A Biblioteca Digital de Teses e Dissertações (BDTD) da Universidade Federal do Rio de Janeiro (UFRJ) integra a Base Minerva ${ }^{1}$, o catálogo online de pesquisa bibliográfica que oferece vários recursos para auxiliar na busca e recuperação das informações e na organização dos resultados encontrados.

Este trabalho tem por objetivo mapear a produção científica dos últimos dez anos dos discentes dos Programas de pós-graduação do Instituto de Matemática (IM) da UFRJ.

Dentre as diversas produções científicas as teses e dissertações destacam-se por serem produzidas em Programas de pós-graduação e avaliadas por pares em bancas de conhecimento reconhecido (MORAES; OLIVEIRA, 2010).

\section{REVISÃO DE LITERATURA}

Segundo Leite Filho (2008) o papel fundamental da produção do conhecimento é o de servir de referência para os estudantes. Neste contexto temos os Programas de pós-graduação, pois é a partir deles que há a formação de pesquisadores, professores, mestres e doutores que irão contribuir para a produção do conhecimento científico.

Os Programas de pós-graduação stricto sensu ${ }^{2}$ oferecidos pelo IM são os seguintes: Matemática, Ensino de Matemática, Estatística, Mestrado Profissional em Matemática em Rede Nacional (PROFMAT), Informática e História das Ciências e das Técnicas e Epistemologia. A Biblioteca Leopoldo Nachbin, também conhecida como a Biblioteca do $\mathrm{IM}^{3}$, abriga em seu acervo as teses e dissertações dos Programas de pós-graduação do IM, com exceção dos trabalhos dos Programas de Informática e História das Ciências e das Técnicas e Epistemologia. Por razões de espaço físico, a partir do ano de 2019 não estão sendo depositados na biblioteca os exemplares impressos dos trabalhos, apenas seu formato digital.

Em todo o mundo o movimento a favor do acesso aberto das produções científicas têm mobilizado pesquisadores e organizações, portanto no ano de 2004 passou a ser obrigatório o depósito em formato digital das teses e dissertações da universidade, sendo disponibilizadas na Base Minerva. $\mathrm{O}$ número de teses e dissertações disponibilizadas na BDTD/UFRJ não corresponde ao volume das produções já geradas na pós-graduação do IM. Diante disso, as teses e dissertações anteriores ao ano de 2004 e que não estão disponíveis online na Base Minerva, estão sendo digitalizadas de forma gradual, a fim de que toda a produção acadêmica possa ser consultada remotamente.

A Central de Memória Acadêmica (CMA) é a Unidade responsável pela guarda dos exemplares impressos dos trabalhos da pós-graduação da universidade. Vale ressaltar que o Repositório Institucional da UFRJ, denominado Pantheon ${ }^{4}$, também disponibiliza as teses e dissertações da comunidade acadêmica, além de outros trabalhos.

Sobre as teses e dissertações e sua importância para a formação do patrimônio intelectual podemos afirmar que:

A produção acadêmica dos cursos de pós-graduação, especificamente de dissertações e teses, é importante veículo para a divulgação do conhecimento científico e para a geração de novos conhecimentos, contribuindo para a formação do nosso patrimônio intelectual (BOTTARI; SILVA, 2011, p. 88).

Ainda sobre as teses e dissertações, Moraes e Oliveira (2010, p. 79) enfatizam que:

Elas são indicadores de avaliação da produção científica de uma área e de um país e servem como subsídio para a política de ensino e pesquisa nacional. Observando-as é possível localizar as áreas do conhecimento em expansão e as lacunas de pesquisa tanto institucional como nacional.

\footnotetext{
${ }^{1}$ Disponível em: https://minerva.ufrj.br

${ }^{2}$ Disponível em: http://www.im.ufrj.br/index.php/pt/ensino/pos-graduacao/pos-graduacao-do-im

${ }^{3}$ Disponível em: http://www.im.biblioteca.ufrj.br

${ }^{4}$ Disponível em: https://pantheon.ufrj.br
} 
Sendo assim, acredita-se que a disponibilização online deste acervo na BDTD/UFRJ contribuirá para a democratização do acesso e a disseminação da produção intelectual e científica da universidade.

\section{METODOLOGIA}

Este estudo caracteriza-se por uma pesquisa descritiva, cuja abordagem é quantitativa, pois visa apresentar as teses e dissertações produzidas pelos discentes dos cursos de pós-graduação do IM.

O universo da pesquisa foram as teses e dissertações e a amostra foi delimitada pelo período dos últimos dez anos, ou seja, de 2010 à 2019.

O instrumento utilizado para a coleta dos dados foi a Base Minerva através de uma busca bibliográfica, observando-se apenas as teses e dissertações pertencentes ao acervo da Biblioteca do IM. Para a coleta foi realizada uma pesquisa no campo "Busca Avançada", utilizando o filtro "Programa de Pós", seguido do nome do Programa + o operador booleano NOT + o termo "Rede Nacional" e "Ensino", a fim de se evitar registros duplicados. Esta foi uma limitação encontrada para a pesquisa, cuja amostragem por ser intencional, pôde recuperar registros duplicados. A Base utilizada foi a de Teses e Dissertações, com intervalo de ano a ano, ou seja, cada ano foi pesquisado um por um, com todos os idiomas selecionados.

\section{RESULTADOS}

Após a realização da pesquisa na Base Minerva com os critérios descritos anteriormente, foram obtidos os seguintes resultados para os trabalhos dos cursos de pós-graduação do IM (Quadro1):

Quadro 1 - Número de teses e dissertações registradas na Base Minerva nos últimos dez anos

\begin{tabular}{|lccccccccccc|c|}
\hline $\begin{array}{l}\text { Programas de } \\
\text { pós-graduação }\end{array}$ & $\mathbf{2 0 1 0}$ & $\mathbf{2 0 1 1}$ & $\mathbf{2 0 1 2}$ & $\mathbf{2 0 1 3}$ & $\mathbf{2 0 1 4}$ & $\mathbf{2 0 1 5}$ & $\mathbf{2 0 1 6}$ & $\mathbf{2 0 1 7}$ & $\mathbf{2 0 1 8}$ & $\mathbf{2 0 1 9}$ & TOTAL \\
\hline $\begin{array}{l}\text { Ensino de } \\
\text { Matemática }\end{array}$ & 4 & 7 & 4 & 12 & 6 & 5 & 5 & 4 & 8 & 10 & $\mathbf{6 5}$ \\
\hline Matemática & 8 & 16 & 7 & 25 & 24 & 21 & 26 & 26 & 16 & 8 & $\mathbf{1 7 7}$ \\
\hline Estatística & 5 & 6 & 8 & 13 & 8 & 13 & 4 & 9 & 10 & 6 & $\mathbf{8 2}$ \\
\hline PROFMAT & 0 & 0 & 0 & 3 & 5 & 5 & 6 & 14 & 11 & 3 & $\mathbf{4 7}$ \\
\hline TOTAL & $\mathbf{1 7}$ & $\mathbf{2 9}$ & $\mathbf{1 9}$ & $\mathbf{5 3}$ & $\mathbf{4 3}$ & $\mathbf{4 4}$ & $\mathbf{4 1}$ & $\mathbf{5 3}$ & $\mathbf{4 5}$ & $\mathbf{2 7}$ & $\mathbf{3 7 1}$ \\
\hline MÉDIA & & & & & & & & & & & $\mathbf{9 2 , 7 5}$ \\
\hline
\end{tabular}

Fonte: Elaborado pela autora de acordo com a Base Minerva da UFRJ. Acesso em: 28 dez. 2020.

A seguir temos o crescimento da produção acadêmica do IM nos últimos dez anos de acordo com os seus Programas de pós-graduação representados na legenda (Gráfico 1):

\footnotetext{
${ }^{5}$ O PROFMAT teve seu início após os outros cursos do IM, por isso de 2010 a 2012 não há produção.
} 
Gráfico 1 - Evolução da produção discente do IM nos últimos dez anos

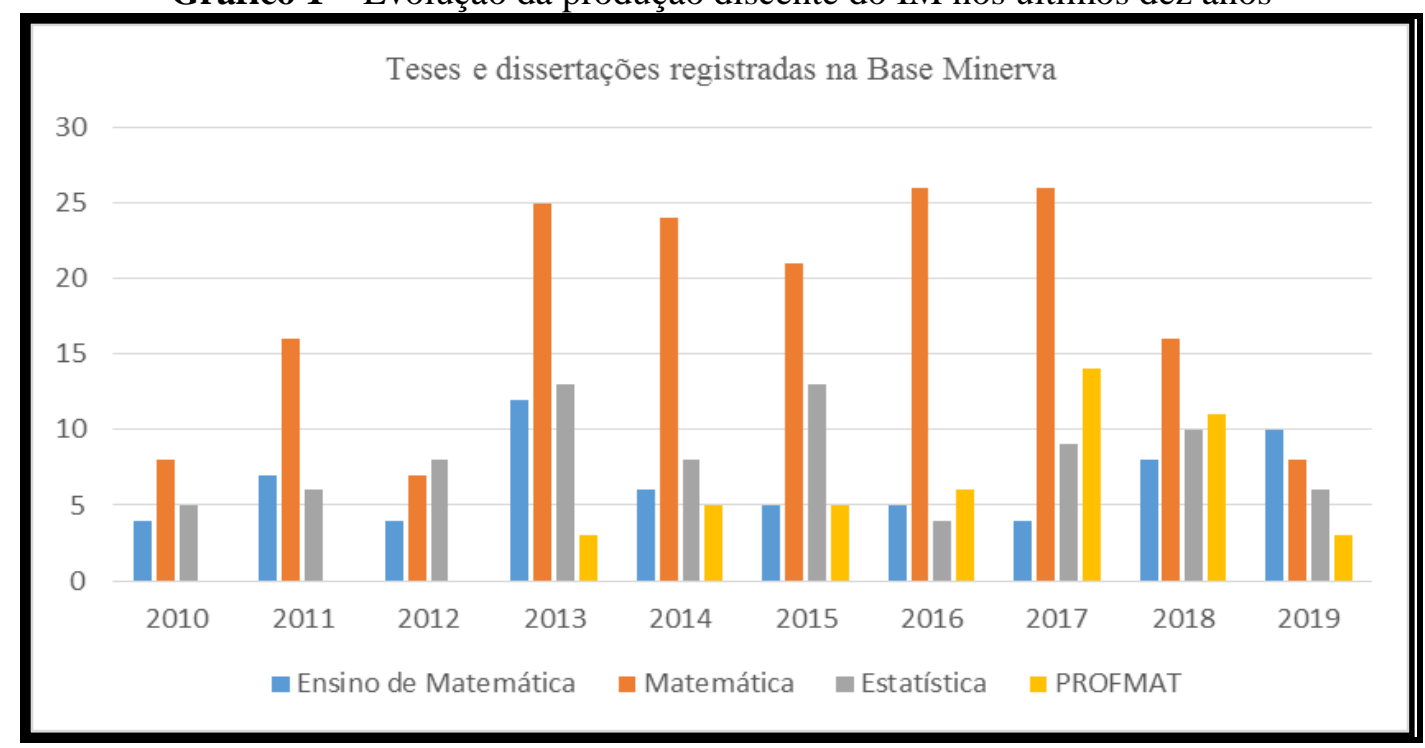

Fonte: Elaborado pela autora conforme a Base Minerva da UFRJ.

Quanto aos Programas da pós-graduação verificou-se que a produtividade científica dos últimos dez anos, ou seja, que a maioria das teses e dissertações são oriundas da Matemática e da Estatística, seguida do Ensino de Matemática e do PROFMAT. Já o Ensino de Matemática obteve sua maior produção em 2013 e desde então vem mantendo o mesmo nível.

O Programa de Matemática obteve um índice de aumento na sua produção no decorrer dos anos e foi mantendo-se ao longo dos anos. Já a Estatística, sofreu um aumento e uma ligeira queda na produção. E por fim, o PROFMAT, vem aumentando sua produção ao longo dos anos desde sua criação.

Quanto ao anos verificados observou-se que os anos de 2013 e 2017 foram os de maior produção científica, agrupando-se os quatro programas de ensino. Concluiu-se que a produção média dos programas foi de 92,75 no período de tempo analisado. É importante ressaltar que alguns trabalhos referentes ao ano de 2019 ainda não foram depositados na biblioteca, portanto podendo interferir nos resultados.

\section{CONSIDERAÇÕES FINAIS}

O objetivo deste estudo foi analisar, sob o ponto de vista da bibliometria, a produção científica dos últimos dez anos dos Programas de pós-graduação do IM.

É importante ressaltar a contribuição da BDTD da UFRJ para a divulgação da produção científica e para a democratização do acesso ao conhecimento acadêmico.

Também podemos observar a evolução e o crescimento da produção científica discente no decorrer dos últimos dez anos e considerando-se as limitações da pesquisa foi possível observar que seus resultados podem contribuir para o entendimento da produção científica na área de Matemática e afins e também para a produção discente da UFRJ.

\section{REFERÊNCIAS}

BOTTARI, Christina Thereza R.; SILVA, Neusa Cardim da. Biblioteca Digital de Teses e Dissertações da UERJ: desafios e oportunidades. Informação \& Informação, Londrina, v. 16, n.1, p. 88-101, jan./jun. 2011. 
LEITE FILHO, Geraldo Alemandro. Padrões de produtividade de autores em periódicos e congressos na área de contabilidade no Brasil: um estudo bibliométrico. Revista de Administração

Contemporânea, Curitiba, v. 12, n. 2, abr./jun. 2008.

MORAES, Alice Ferry de; OLIVEIRA, Telma Maria de. Experiências relacionadas ao levantamento de teses e dissertações. Informação \& Sociedade: Estudos, João Pessoa, v.20, n.1, p. 73-81, jan./abr. 2010. 